\title{
Intraoperative monitoring for tethered cord surgery: an update
}

\author{
Karl F. Kothbauer, M.D., and KLaus NovaK, M.D. \\ Divisions of Pediatric Neurosurgery, and Intraoperative Neurophysiology, Institute for Neurology \\ and Neurosurgery, Beth Israel Medical Center, New York, New York
}

\begin{abstract}
Object. Intraoperative neurophysiological recording techniques have found increasing use in neurosurgical practice. The development of new recording techniques feasible while the patient receives a general anesthetic have improved their practical use in a similar way to the use of digital recording, documentation, and video technology. This review intends to provide an update on the techniques used and their validity.

Methods. Two principal methods are used for intraoperative neurophysiological testing during tethered cord release. Mapping identifies functional neural structures, namely nerve roots, and monitoring provides continuous information on the functional integrity of motor and sensory pathways as well as reflex circuitry. Mapping is performed mostly by using direct electrical stimulation of a structure within the surgical field and recording at a distant site, usually a muscle. Sensory mapping can also be performed with peripheral stimulation and recording within the surgical site. Monitoring of the motor system is achieved with motor evoked potentials. These are evoked by transcranial electrical stimulation and recorded from limb muscles and the external anal sphincter. The presence or absence of muscle responses are the parameters monitored. Sensory potentials evoked by tibial or pudendal nerve stimulation and recorded from the dorsal columns via an epidurally inserted electrode and/or from the scalp as cortical responses are used to access the integrity of sensory pathways. Amplitudes and latencies of these responses are then interpreted. The bulbocavernosus reflex, with stimulation of the pudendal nerve and recording of muscle responses in the external anal sphincter, is used for continuous monitoring of the reflex circuitry. Presence or absence of this response is the pertinent parameter that is monitored.

Conclusions. Intraoperative neurophysiology provides a wide and reliable set of techniques for intraoperative identification of neural structures and continuous monitoring of their functional integrity.
\end{abstract}

\section{KEY WORDS • tethered cord • intraoperative • neurophysiological monitoring • evoked potentials $\bullet$ mapping}

Intraoperative neurophysiological monitoring has long been performed by neurosurgeons during procedures to release the tethered spinal cord. ${ }^{5,15,17,18,25,26}$ Urological $^{1}$ and electrophysiological techniques ${ }^{3,7,17,26}$ have been applied to monitor procedures involving the conus and the cauda equina: continuous recording of EMG activity in sphincter muscles,${ }^{7,8}$ recording of CMAPs from leg ${ }^{17,26}$ and sphincter $r^{7,17,26}$ muscles to electrical stimulation in the operating field, direct SSEP recording from roots to electrical stimulation in the periphery, ${ }^{4}$ and measurement of sphincter tone and bladder pressure. ${ }^{20,26}$ The use of these techniques in the context of surgery for dysraphism has been presented in various formats in the past in updates by several authors. ${ }^{12,15,23-26}$

During surgery for release of a tethered cord it may be necessary to cut the terminal filum, to dissect broad-based scar tissue that envelops the conus medullaris and/or the

Abbreviations used in this paper: $\mathrm{BCR}=$ bulbocavernosus reflex; CMAP = compound muscle action potential; EMG = electromyography; $\mathrm{MEP}=$ motor evoked potential; SSEP = somatosensory evoked potential. cauda equina nerve roots, to cut numerous fibrous bands that hold the conus rigidly in its position, or it may be necessary to resect partially a lipoma located at or within the conus. Thus, correct distinction between functional nervous tissue and fibrous bands is essential in these situations to avoid postoperative sensorimotor deficits and sphincter dysfunction. Direct stimulation of these structures in the surgical field or direct recording from them has improved this distinction beyond morphological recognition under the surgical microscope and reliance on the surgeon's experience. By using the mapping concept all functional neural structures of the lumbosacral region can be correctly identified and thus preserved.

During untethering procedures the conus or individual nerve roots may be inadvertently damaged by traction, compression, or coagulation. Many times this damage is reversible if detected early and if its cause is corrected. To detect such potentially reversible damage the functional integrity of the involved pathways has to be assessed continuously with monitoring.

Monitoring and mapping of the cauda equina and conus medullaris includes the simultaneous application of a 
number of modalities. It therefore requires the availability of a powerful multichannel recording system. All recordings in our institution are obtained with the Axon Sentinel-4 EP analyzer (Axon Systems Inc., Hauppauge, NY), which is equipped with dedicated software for controlling transcranial stimulation paradigms.

\section{ANESTHESIA}

Anesthetic management, which allows intraoperative MEP monitoring, consists of a constant infusion of propofol (usually in a dose of $\sim 100-150 \mu \mathrm{g} / \mathrm{kg} / \mathrm{min}$ ) and fentanyl (usually $\sim 1 \mu \mathrm{g} / \mathrm{kg} / \mathrm{hr}$ ). The use of propofol as an anesthetic with MEP monitoring has been reported with various stimulation techniques. ${ }^{9,11,27,29}$ Halogenated anesthetics cannot be used. ${ }^{29}$ Short-acting muscle relaxants are given for intubation but not thereafter. Management of anesthetics during operations in which intraoperative neurophysiolgoical monitoring has been used has recently been extensively reviewed. ${ }^{27}$

\section{MAPPING}

Mapping is used to identify electrophysiologically motor and sensory nerve roots and thereby distinguish them from scar tissue and fibrous bands. This allows safe transection of fibrous tethering structures while ensuring that optimal untethering is accomplished.

\section{Motor Root Mapping}

To identify motor nerve roots of the cauda equina intraoperatively, structures to be identified are directly stimulated with a hand-held monopolar stimulator or with a bipolar stimulation forceps. Bilateral recording from segmental target muscles reveals the EMG responses in muscles supplied by the stimulated nerve root. Recording from segmental target muscles for all lumbosacral myotomes (Table 1) ensures that all pertinent motor roots are covered. If a nerve root is stimulated, the corresponding muscle on the corresponding side will show a CMAP. These responses are obtained instantly, thus the mapping information is available without any delay (Video Clip 1).

Click here to view Video Clip 1. The terminal filum is exposed and nerve roots of the cauda equina are stimulated using a monopolar hand-held stimulator. Sacral nerve roots are identified by this technique.

\section{Sensory Root Mapping}

After direct stimulation of a sensory nerve root in the cauda equina one expects a CMAP in the corresponding

TABLE 1

Segmental muscle recording sites

\begin{tabular}{cl}
\hline \hline Spinal Level & \multicolumn{1}{c}{ Muscle(s) } \\
\hline L-2 & gracilis, pectineus \\
L-3 & quadriceps, adductors \\
L-4 & quadriceps, tibialis anterior \\
L-5 & tibialis anterior, extensor hallucis longus \\
S-1 & gastrocnemius, biceps femoris, gluteus maximus \\
S-2 & soleus, external anal sphincter \\
S-3 & abductor hallucis, external anal sphincter \\
S-4 & external anal sphincter \\
\hline
\end{tabular}

segmental muscles, which is generated in the conus the same way as the H-reflex. If the sacral dorsal roots (S2-4) are stimulated, an anal sphincter response equivalent to the BCR can be recorded. Even if the afferents are intact, the efferent fibers or the corresponding segment in the spinal cord may be impaired, and therefore, there may be no motor response. Stimulation of this presumed sensory root with SSEP parameters and recording either from the epidural electrode or from the cortex may identify the root as sensory. ${ }^{15}$

Similarly a structure can also be identified as a sensory nerve root by stimulation at the pudendal nerve and recording of a sensory nerve action potential, ${ }^{4}$ a technique originally developed for pudendal afferent mapping to minimize sphincter dysfunction resulting from surgery for selective posterior rhizotomy. ${ }^{6}$ The pudendal nerve branches are electrically stimulated with surface electrodes that are attached over the dorsal surface of the penis or clitoris (single stimuli of $200 \mu \mathrm{sec}$ duration, intensity $20 \mathrm{~mA}$, stimulation rate $13.3 \mathrm{~Hz}$ ). Based on experience in performing rhizotomy it has become apparent that the distribution of pudendal afferent nerve fibers to S2-4 posterior roots bilaterally is frequently not symmetrical or evenly distributed over these three segmental roots. In approximately $7 \%$ of individuals one single dorsal root on one side was found to carry all pudendal afferents. ${ }^{4}$ Injury to this single root would presumably result in significant pudendal afferent dysfunction and consequently in a loss of sphincter function. It must be assumed that the distribution of pudendal afferents in patients with a dysraphic condition is at least as asymmetrical and unevenly distributed as in patients with spasticity. Therefore the pinpointing of a structure as a root and as a posterior root may at times be essential. Sensory mapping requires a degree of signal averaging; therefore, the mapping information is not instantly available but with a short delay of approximately 30 seconds to 2 minutes, depending on recording quality and signal/noise ratio.

\section{MONITORING}

Monitoring provides continuous information about the functional integrity of the monitored system: motor, sensory, or reflex circuitry.

\section{Muscle MEPs}

Muscle MEPs are evoked using transcranial electrical stimulation with a multipulse or train stimulation technique, ${ }^{10,12-14,21,28}$ and recorded from the same segmental target muscles chosen for mapping (Figs. 1-3).

Motor sensory evoked potentials provide immediate information about the functional integrity of the segments monitored. The presence or absence of recordable potentials with stimulation intensities usually not exceeding $200 \mathrm{~mA}$ is the pertinent parameter monitored. Adequate information for reliable interpretation of changes in stimulation threshold intensities is not available. The presence of muscle MEPs correlates with preserved motor control in all instances. Intraoperative loss of muscle MEPs correlates with a postoperative motor deficit. Unlike in surgery for more proximal intramedullary spinal cord tumors ${ }^{16}$ there is, at this time, no clearly defined neurophysiological concept of reversible damage. Therefore it 


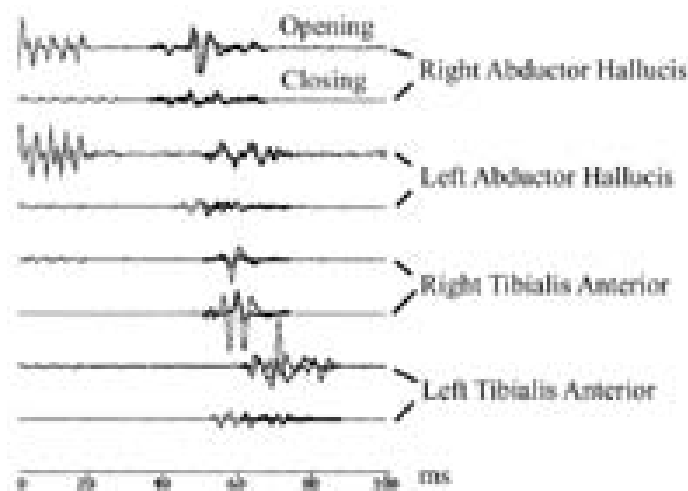

Fig. 1. Baseline and closing recordings of MEPs obtained from the abductor hallucis and the tibialis anterior muscles bilaterally. All requisite recordings are present, indicating intact motor control. MS = microsecond .

is imperative to preserve MEPs throughout the procedure. Loss of muscle MEPs in cauda equina or conus surgery may indicate a complete lower motor neuron lesion, producing a postoperative motor deficit with presumably little tendency to recover. After loss of a segmental lower motor neuron, distal plasticity with a secondary increase in the size of motor units supplying the affected muscle from neighboring segments could result in long-term improvement of motor deficits. Unlike monitoring for spinal cord surgery proximal to the conus, monitoring of epidural MEPs (D-wave monitoring ${ }^{19}$ ) is not possible during tethered cord release, because the corticospinal tract ends in the conus and the structures at risk are in the conus or distal to it (Video Clip 2).

Click here to view Video Clip 2. Bulbocavernosus reflex recordings and MEPs are visualized in direct documentation of video-input from the surgical microscope and direct real-time correlation of the recordings.

\section{Somatosensory Evoked Potentials}

Somatosensory evoked potentials, after stimulation of the tibial nerve at the ankle or knee and recording both from the spinal cord with an epidurally placed elec-
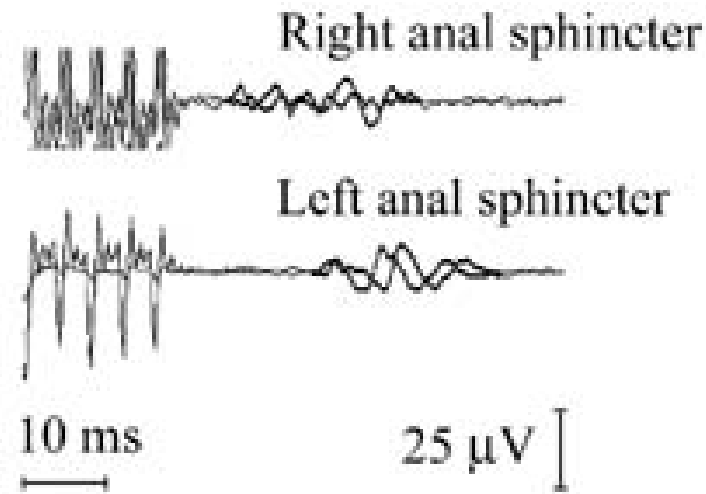

Fig. 2. Tracings showing MEPs recorded from the external anal sphincter after transcranial electrical stimulation. These recordings are obtained in the same way as MEPs in limb muscles.

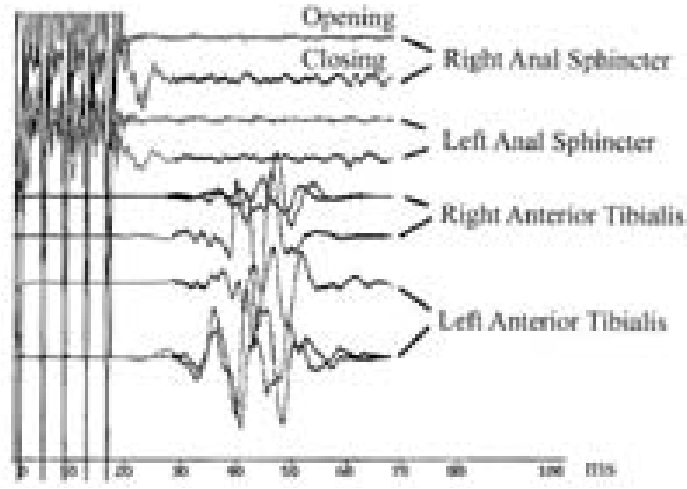

Fig. 3. Baseline MEPs obtained in a patient with preoperative sphincter dysfunction. This patient required clean intermittent catheterization before surgery. There are no MEPs present from the sphincter. The limb muscle MEPs are present. The presence of lower-extremity MEPs correlated with intact motor function; the absence of anal sphincter MEPs correlated with lack of sphincter control.

trode (Fig. 4) and from the cortex, can be used to monitor continuously the sensory pathways of L-5 and S- 1 . It is sometimes possible to record spinal and cortical responses to stimulation of the pudendal nerve as well. The preservation of these responses indicates intact functional integrity of the sensory pathways. Their loss or significant deterioration may be indicative of damage to these tracts or corresponding posterior roots. The disadvantages of SSEP monitoring, such as the relatively long averaging time and response fluctuations, however, do apply.

\section{The BCR}

The dorsal penile/clitoral nerve is stimulated via two surface electrodes. In males the electrodes are placed on the dorsum of the penis; in females the cathode is placed over the clitoris and the anode is placed on the adjacent labium on one side. Recordings are obtained from the external anal sphincter muscle with wire or needle electrodes (Fig. 5).

Because the BCR is an oligosynaptic reflex it could be

\section{Spinal Sensory Evoked Potentials}

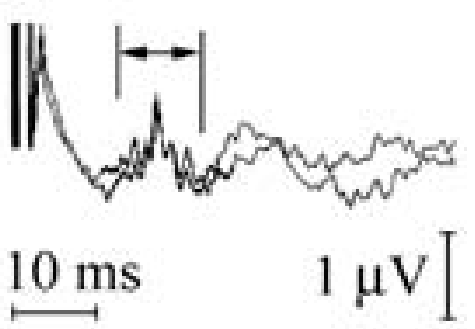

Fig. 4. Spinal SSEPs recorded as a traveling wave directly from the spinal cord at the epiconus level by using an epidurally inserted electrode. 


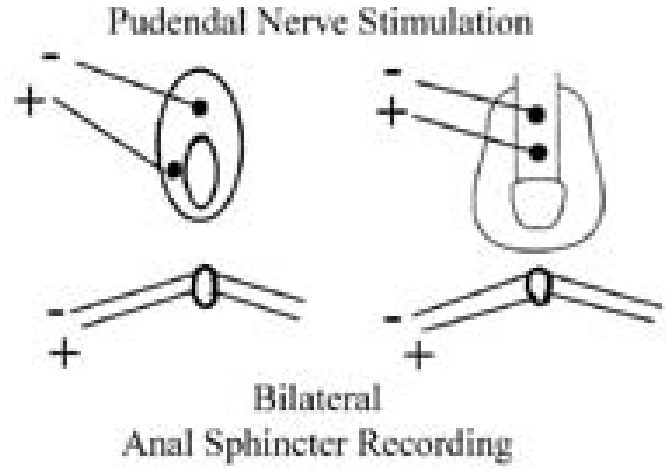

Fig. 5. Schematic representation of the setup used for pudendal nerve stimulation and external anal sphincter recording. This electrode montage is used for BCR recordings, for anal MEP recordings, and for pudendal afferent mapping.

difficult to obtain while the patient is receiving a general anesthetic particularly when volatile anesthetics are used. Therefore the same anesthetic regimen as has been outlined for MEP monitoring is used when BCR monitoring is conducted. Temporal summation is necessary to elicit the BCR under these conditions. This is again achieved by double stimulation or is optimal with a short train of five stimuli (Fig. 6). ${ }^{3}$ The presence of the BCR during surgery indicates intact sphincter control. Intraoperative loss of the BCR indicates at least transient loss of sphincter control; however, intraoperative BCR data and their correlation to long-term sphincter control, sphincter-detrusor muscle dyssynergia, and sexual function still require further investigation (Video Clip 2).

\section{Continuous EMG Monitoring}

All lumbosacral nerve roots can be monitored for peripheral nerve injury by using continuous monitoring of the EMG activity in the aforementioned segmental target muscles. Motor unit potentials and neurotonic discharges are the injury indicators in nerve roots that have been damaged by traction, compression, transection, or thermal injury. ${ }^{2}$ For cauda equina monitoring as well as for brainstem and cranial nerve monitoring the sensitivity and

\section{Bulbocavernosus Reflex}

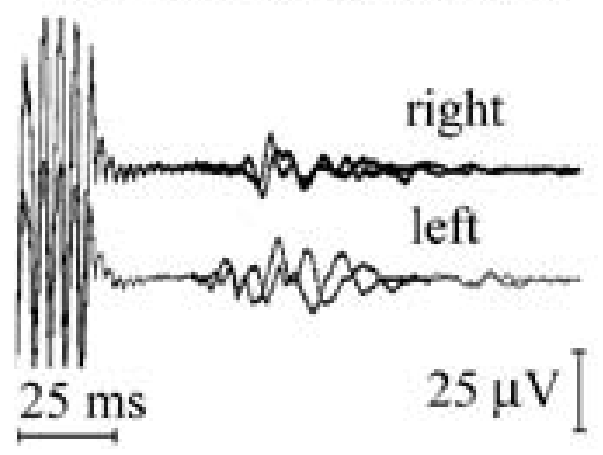

Fig. 6. Bulbocavernosus reflex recordings obtained in a patient with intact sphincter function. specificity of these EMG phenomena are still disputed, and unfortunately the underlying mechanisms are still poorly understood. Nevertheless, there is little doubt that in most instances sustained neurotonic discharges and motor unit potentials indicate some degree of lower motor neuron damage. This phenomenon has been studied in considerable detail for facial nerve monitoring during resection of vestibular schwannomas. A certain type of sustained neurotonic discharge, called A-waves, are indicators of postoperative nerve dysfunction. ${ }^{22}$ It is reasonable to assume that similar phenomena may be observed during surgery affecting cauda equina roots; however, in the experience of our group, such discharges have not been observed. It should also be understood that continuous EMG monitoring is not the monitoring of the functional integrity of a pathway but rather an observation of random injury evoked activity. Absence of this activity may not necessarily mean the absence of injury.

\section{CONCLUSIONS}

During surgery for release of a tethered cord intraoperative neurophysiological mapping provides accurate and practical means for identifying neural structures, which may be difficult to distinguish from fibrous tissue based on morphological approach alone. Both motor and sensory roots can be mapped. Direct stimulation in the surgical field is the most straightforward and practical technique for this purpose.

Continuous MEP monitoring provides fast real-time information on the functional integrity of the motor pathways with excellent correlation to clinical outcome. By using BCR, the integrity of the conus reflex circuitry can be monitored intraoperatively; however, the prognostic value of BCR monitoring still has to be determined. Monitoring of SSEPs provides information about the sensory pathways with the expected short time delay inevitable for a technique that requires signal averaging.

\section{Acknowledgments}

We are indebted to Vedran Deletis, Fred J. Epstein, Sedat Ulkatan, and the members of the Division for Intraoperative Neurophysiology at the Institute for Neurology and Neurosurgery at the Beth Israel Medical Center, New York.

\section{References}

1. Bradley WE, Timm GW: Combined electromyographic and gas urethral pressure profilometry. J Urol 115:433-434, 1976

2. Daube JR: Intraoperative monitoring of cranial motor nerves, in Schramm J, Møller AR (eds): Intraoperative Neurophysiologic Monitoring in Neurosurgery. Berlin: Springer-Verlag, 1991, pp 246-267 (Reference unverified)

3. Deletis V, Vodusek DB: Intraoperative recording of the bulbocavernosus reflex. Neurosurgery 40:88-93, 1997

4. Deletis V, Vodusek DB, Abbott R, et al: Intraoperative monitoring of the dorsal sacral roots: minimizing the risk of iatrogenic micturition disorders. Neurosurgery 30:72-75, 1992

5. Haldeman S, Bradley WE, Bhatia NN, et al: Pudendal evoked responses. Arch Neurol 39:280-283, 1982

6. Huang JC, Deletis V, Vodusek DB, et al: Preservation of pudendal afferents in sacral rhizotomies. Neurosurgery 41:411-415, 1997

7. James HE, Mulcahy JJ, Walsh JW, et al: Use of anal sphincter 
electromyography during operations on the conus medullaris and sacral nerve roots. Neurosurgery 4:521-523, 1979

8. James HE, Williams J, Brock W, et al: Radical removal of lipomas of the conus and cauda equina with laser microneurosurgery. Neurosurgery 15:340-343, 1984

9. Jellinek D, Jewkes D, Symon L: Noninvasive intraoperative monitoring of motor evoked potentials under propofol anesthesia: effects of spinal surgery on the amplitude and latency of motor evoked potentials. Neurosurgery 29:551-557, 1991

10. Jones SJ, Harrison R, Koh KF, et al: Motor evoked potential monitoring during spinal surgery: responses of distal limb muscles to transcranial cortical stimulation with pulse trains. Electroencephalogr Clin Neurophysiol 100:375-383, 1996

11. Kalkman CJ, Drummond JC, Ribberink AA, et al: Effects of propofol, etomidate, midazolam, and fentanyl on motor evoked responses to transcranial electrical or magnetic stimulation in humans. Anesthesiology 76:502-509, 1992

12. Kothbauer K, Deletis V, Epstein FJ: Intraoperative neurophysiological monitoring, in Crockard A, Hayward R, Hoff JT (eds): Neurosurgery: The Scientific Basis of Clinical Practice, ed 3. Oxford: Blackwell Science, 2000, Vol 2, pp 1041-1057

13. Kothbauer K, Deletis V, Epstein FJ: Intraoperative spinal cord monitoring for intramedullary surgery: an essential adjunct. Pediatr Neurosurg 26:247-254, 1997

14. Kothbauer K, Deletis V, Epstein FJ: Motor evoked potential monitoring for spinal cord tumor surgery. J Neurosurg 88: 403A, 1998 (Abstract)

15. Kothbauer K, Schmid UD, Seiler RW, et al: Intraoperative motor and sensory monitoring of the cauda equina. Neurosurgery 34:702-707, 1994

16. Kothbauer KF, Deletis V, Epstein FJ: Motor-evoked potential monitoring for intramedullary spinal cord tumor surgery: correlation of clinical and neurophysiological data in a series of 100 consecutive procedures. Neurosurg Focus 4 (5):Article 1, 1998

17. Legatt AD, Schroeder CE, Gill B, et al: Electrical stimulation and multichannel EMG recording for identification of functional neural tissue during cauda equina surgery. Childs Nerv Syst 8:185-189, 1992

18. Lueders H, Hahn J, Gurd A, et al: Surgical monitoring of spinal cord function: cauda equina stimulation technique. Neurosurgery 11:482-485, 1982

19. Morota N, Deletis V, Constantini S, et al: The role of motor evoked potentials during surgery for intramedullary spinal cord tumors. Neurosurgery 41:1327-1336, 1997

20. Pang D, Casey K: Use of an anal sphincter pressure monitor during operations on the sacral spinal cord and nerve roots. Neurosurgery 13:562-568, 1983

21. Pechstein U, Cedzich C, Nadstawek J, et al: Transcranial highfrequency repetitive electrical stimulation for recording myogenic motor evoked potentials with the patient under general anesthesia. Neurosurgery 39:335-344, 1996

22. Romstöck J, Strauss C, Fahlbusch R: Continuous electromyography monitoring of motor cranial nerves during cerebellopontine angle surgery. J Neurosurg 93:586-593, 2000

23. Sala F, Krzan M, Deletis V: Intraoperative neurophysiological monitoring in pediatric neurosurgery: why, when, how? Childs Nerv Syst 18:264-287, 2002

24. Sala F, Krzan MJ, Epstein FJ, et al: Specificity of neurophysiological monitoring of the lumbosacral nervous system during tethered cord release: a preliminary report. Childs Nerv Syst 15:426, 1999 (Abstract)

25. Schmid UD, Gall C, Schröck E, et al: Funktionskontrollierte Neurochirurgie. Nervenarzt 66:582-595, 1995

26. Shinomiya K, Fuchioka M, Matsuoka T, et al: Intraoperative monitoring for tethered spinal cord syndrome. Spine 16: 1290-1294, 1991

27. Sloan TB: Anesthesia and motor evoked potential monitoring, in Deletis V, Shils J (eds): Neurophysiology in Neurosurgery: A Modern Intraoperative Approach. Amsterdam: Academic Press, 2002, Vol 1, pp 451-474

28. Taniguchi M, Cedzich C, Schramm J: Modification of cortical stimulation for motor evoked potentials under general anesthesia: technical description. Neurosurgery 32:219-226, 1993

29. Taniguchi M, Nadstawek J, Langenbach U, et al: Effects of four intravenous anesthetic agents on motor evoked potentials elicited by magnetic transcranial stimulation. Neurosurgery 33: 407-415, 1993

Manuscript received December 15, 2003.

Accepted in final form January 13, 2004.

Address reprint requests to: Karl F. Kothbauer, M.D., Division of Neurosurgery, Department of Surgery, Kantonsspital Luzern, Postfach, CH-6000 Luzern 16, Switzerland. email: kkothbau@excite. com, neuro-luzern@excite.com, kkothbau@bethisraelny.org. 